\title{
Caracterización de la crianza no tecnificada de cerdos en el parque porcino del distrito de Villa el Salvador, Lima-Perú.
}

Characterization of non-tech breeding pigs in a pig Park district of Villa El Salvador in Lima-Perú

Rosa Morales ${ }^{1}$, Monica Rebatta ${ }^{2}$, Juan Lucas² Javier Mateo ${ }^{3}$, Daphne Ramos ${ }^{2}$

\section{RESUMEN}

Objetivo: Identificar las condiciones de manejo e infraestructura en la crianza no tecnificada de cerdos en una zona peri-urbanas de Lima. Metodología: Se encuesto a 51 productores. Se tomaron datos como información demográfica y características del sistema productivo. Resultados y Conclusiones: La edad promedio de los productores fue de 51.3 años (rangos de 19 a 74 años) y el tiempo promedio que dedicaban a la actividad fue de 10.8 años (rangos de 01 a 45 años). Se registró como propiedad un total de 1401 animales (290 marranas, 34 verracos, 622 gorrinos y 455 lechones). El número de lechones nacidos por cada parto fue de 10.1 y la cantidad de nacimientos por año por productor fue de 94. Las principales causas de morbi-mortalidad fueron los problemas diarreicos y el cólera porcino. El 86.3\% cocina los alimentos que consumen los animales. El $64.7 \%$ no recibe asesoría profesional, el $89.9 \%$ aplica vacunación contra el cólera y el $72.5 \%$ no lleva ningún tipo de registro. El modo predominante de comercialización de los cerdos es la venta en pie a intermediarios o acopiadores (84\%), donde el cálculo del peso se hace visualmente. La condición de los corrales fue considerada buena solo en 2 casos. La madera es el material predominante en cercos, comederos y bebederos. Los pisos son de tierra. El $86.3 \%$ obtiene agua del camión cisterna, el $86.3 \%$ poseía letrinas y el $57.7 \%$ cuenta con servicio eléctrico. Los resultados del estudio permitieron identificar las principales deficiencias de este proceso productivo y a partir de ellas se pueden aplicar estrategias de intervención para superarlas.

Palabras clave: crianza no tecnificada, parque porcino, porcicultura.

\begin{abstract}
Aim: To identify management and infrastructure deficiencies in the swine non-technified swine breeding in one suburbs of Lima. Methods: 51 Pig producers were surveyed about characteristics of their productive system. Results and Conclusions: The producers average age was 51.3 years (range between 19 to 74 years) and average time dedicated to the activity was 10.8 years (range between 1 to 45 years). They own 1401 animals ( 290 sows, 34 boars, 622 suckling pigs and 455 piglets). The number of piglets per litter was 10.1 and the number of births per year per producer was 94 . Main causes of morbi-mortality were diarrheic problems and hog cholera. From the total of surveyed producers, $86.3 \%$ cook the food for pigs, $64.7 \%$ do not receive professional advice, $89.9 \%$ vaccinate against hog cholera, and $72.5 \%$ do not keep any kind of record. Livestock sale to intermediaries and collectors is the
\end{abstract}

\footnotetext{
${ }^{1}$ Facultad de Medicina Veterinaria y Zootecnia, Universidad Peruana Cayetano Heredia.

${ }^{2}$ Facultad de Medicina Veterinaria, Universidad Nacional Mayor de San Marcos, Perú.

${ }^{3}$ Facultad de Medicina Veterinaria, Universidad de León, España.
} 
predominant way of trading (84\%) and weight approximation is made visually. Pen condition was considered good only in two cases. Predominantly, wood was used for fences, feeders and water trough; and soil for the floor. $86.3 \%$ get water from tanker trucks, $86.3 \%$ have latrines, and $57.7 \%$ have electric service. The results of this study allow us to identify the main deficiencies of this productive process, thus, strategies can be applied in order to overcome them.

Key words: non-technified breeding pigs, porciculture, survey.

\section{INTRODUCCIÓN}

La porcicultura es una actividad importante y creciente para la economía del Perú (Arce et al, 2006). La producción porcina se realiza en tres tipos de crianza: (1) Tecnificada los que cumplen con condiciones de infraestructura especializada, pie de cría con genética avanzada, práctica de rigurosas medidas de bioseguridad, y nivel sanitario adecuado, (2) Semitecnificada, en donde se cumplen medianamente con las condiciones de bioseguridad elementales, infraestructura adaptada al fin, pie de cría con grado de mejoramiento genético intermedio y nivel sanitario aceptable y (3) Crianza casera, como actividad secundaria, complementaria a otras actividades agropecuarias en el medio rural; o una crianza de traspatio, con características de autoconsumo, desarrolladas con mayor frecuencia en zonas suburbanas (Kalinowski, 1996).

En zonas urbanas de latino américa, la cría de cerdos se concentra principalmente en los asentamientos humanos y pueblos jóvenes, barrios populares ubicados en zonas marginales y caracterizados por la precariedad de sus construcciones y la falta de servicios urbanos, y es desarrollada por los clasificadores-criadores y sus familias (Santandreu et al., 2002). Esta actividad genera ganancias importantes que contribuyen a la economía familiar que puede llegar a alcanzar hasta $50,70 \mathrm{y} / \mathrm{o} 90 \%$ en el caso de no contar con un trabajo asalariado (Rivera et al., 2007). Esta crianza también permite diversificar fuentes de ingreso, que permite garantizar la estabilidad económica familiar (Castro y Lozano, 2009)

Los parámetros productivos y reproductivos de los cerdos de crianza no tecnificada son bajos. Así tenemos que la edad al primer servicio es de 8 meses (mínimo 6 y máximo 12 meses), las marranas tienen solamente dos partos al año y una vida reproductiva limitada que va de tres a cinco camadas; asimismo el número de lechones nacidos varía entre 3 y 14 (Zarate, 2006) pero tienen una mortalidad elevada cercana al $50 \%$ (Arce et al, 2006).
Los principales insumos utilizados para la alimentación de los cerdos en orden de importancia son; residuos de restaurantes, casas, mercados, panaderías de distritos aledaños a la zona de crianza y la tripa de pollo que proviene de los centros de acopio de aves y algunas avícolas (Arce et al, 2006); generalmente sin tratamiento térmico y con escaso valor nutricional (Santandreu et al., 2002). Por otro lado, la inversión económica en granjas de traspatio es menor si se compara con los otros tipos de crianza, lo que genera bajos costos fijos, ya que solo se mantienen en el nivel mínimo para operar (Hernández, 2008).

La comercialización es a través de intermediarios los animales se venden en pie y no llevan un control de los pesos por lo que obtienen precios bajos (Arce et al, 2006); en otros casos se benefician los animales y se procesa clandestinamente la carne (embutidos) comercializándose los productos en el mismo lugar o barrios próximos (Santandreu et al., 2002).

Este tipo de crianza está asociada a la seguridad alimentaria de poblaciones vulnerables, y con la presencia de enfermedades de importancia económica y zoonótica (Castro, 2007); independiente de los graves problemas que se pueden producir por la inadecuada gestión de los residuos sólidos y líquidos, deficiencias en el suministro de agua y falta de saneamiento (Ruiz et al., 2010) y el perjuicio a la salud de las personas y medio ambiente, producido por la combustión de los materiales utilizados como combustible en el tratamiento térmico de los alimentos (Rodríguez et al, 2003; Fernández y Sánchez, 2007).

Debido al impacto de este tipo de crianza sobre la salud pública, es necesario apoyar la paulatina tecnificación de la crianza de cerdos, a través de la promoción de capacitaciones en términos normativos, manejo sanitario, reproductivo y alimenticio, y mercadeo. Para poder desarrollar esto se requiere una evaluación de la línea basal a fin de identificar las principales deficiencias de este proceso productivo y diseñar estrategias específicas para superarlas. Por lo que, el objetivo del estudio fue caracterizar la crianza 
de cerdos en granjas no tecnificadas en la zona de Lomo de Corvina en el distrito de Villa el Salvador en Lima, Perú.

\section{MATERIALES Y METODOS}

El estudio observacional descriptivo de corte transversal se realizó en la Facultad de Medicina Veterinaria y Zootecnia de la Universidad Peruana Cayetano Heredia, utilizando los datos obtenidos por una encuesta realizada en la zona agropecuaria del distrito de Villa El Salvador en la provincia de Lima, región Lima.

La población objetivo y el tamaño de muestra, estuvo conformado por todas las encuestas realizadas durante el año 2010 a los criadores ubicados en el Parque Porcino Lomo de Corvina.

Las encuestas contenían información demográfica de la zona y facilitaba la caracterización de las condiciones de crianza de los porcinos. El mismo constó de la siguiente información: (a) Datos generales del entrevistado, (b) Información acerca de las personas que laboran en la granja y de las personas que dependen económicamente de este sistema productivo y (c) caracterización del sistema productivo porcino (tenencia de animales por categoría, manejo sanitario, reproductivo y alimenticio, mercadeo, descripción de las instalaciones y equipos utilizados en la crianza).

El procesamiento de datos se realizó con el programa SPSS 15.0 usándose la estadística descriptiva para resumir la información.

\section{RESULTADOS}

El estudio involucró a 51 productores de cerdos de la zona de Lomo de Corvina, con una edad promedio de $51.3 \pm 14.0$ años. El 51.0\% era del sexo masculino y el $49 \%$ de sexo femenino. En cuanto a educación el 51\% tenía educación primaria, $27.5 \%$ secundaria,
$15.7 \%$ superior y la diferencia no contestó la pregunta. El tiempo promedio que los productores han estado dedicados a la crianza de cerdos fue de $10.8 \pm 8.2$ años.

En cuanto al ingreso mensual familiar, el $43.1 \%$ de los encuestados mencionó tener un ingreso de hasta 650 nuevos soles, el $25.5 \%$ entre 651 y 1000 soles, $25.5 \%$ entre 1001 a 1500 soles y solo el $5.9 \%$ tenía un ingresos mayor a 1500 nuevos soles.

Se registró un total de 1401 animales. En el cuadro 1 se muestra la distribución de los animales según la categoría productiva. Asimismo, en el Cuadro 2 aparece el número de lechones y de gorrinos a la venta según el tamaño del predio.

El alimento es ofrecido a los animales de diferentes maneras así tenemos que el $86.3 \%$ de los productores cocina los alimentos (sopa) y el $13.7 \%$ restante lo ofrece crudo ya sea puro o mezclado con afrecho. La cocción de los alimentos se realiza básicamente usando leña como combustible y los recipientes usados fueron cilindros $(80 \%)$, ollas (4\%) o ambos en los restantes.

El cuadro 3 muestra la composición del alimento que se ofrece a los animales de acuerdo a la etapa productiva. El $97.9 \%$ de los productores no sabe cuánto peso ganan sus animales por $\mathrm{Kg}$. de alimento ofrecido. La inversión de dinero reportada para la alimentación de los animales al día fue en promedio de $24 \pm 19.4$ soles.

En cuanto a la ayuda profesional para la crianza tenemos que el $64.7 \%$ de los criadores indico que no recibe, el 11.8\% recibe ayuda una vez al mes; el 13.7\% 2 o 3 veces al mes, $3.9 \%$ más de 3 veces al mes y $5.9 \%$ recibe ayuda esporádicamente. La aplicación de la vacuna de cólera porcino es realizada por el $89.9 \%$ de los encuestados y la vacuna contra septicemia es aplicada por el $51 \%$.

Cuadro 1. Número total de animales según Categoría Productiva. Lomo de Corvina, Villa el Salvador - Lima, Perú. 2010

\begin{tabular}{llllllc}
\hline $\begin{array}{l}\text { Categoría } \\
\text { Productiva }\end{array}$ & $\begin{array}{l}\text { Número de } \\
\text { Propietarios }\end{array}$ & $\begin{array}{l}\text { Total de } \\
\text { animales }\end{array}$ & Media & $\begin{array}{l}\text { Desviación } \\
\text { estándar }\end{array}$ & $\begin{array}{l}\text { Rango } \\
\text { Mínimo }\end{array}$ & $\begin{array}{c}\text { Rango } \\
\text { Máximo }\end{array}$ \\
\hline Marranas & 43 & 290 & 6.7 & 4.5 & 1 & 17 \\
Verracos & 25 & 34 & 1.4 & 0.6 & 1 & 3 \\
Gorrinos & 39 & 622 & 16 & 18 & 3 & 90 \\
Lechones & 35 & 455 & 13 & 9.3 & 2 & 40 \\
\hline
\end{tabular}


Cuadro 2. Distribución del número de animales a la venta según categoría productiva y número de marranas. Lomo de Corvina, Villa el Salvador, Lima 2010

\begin{tabular}{lllll}
\hline $\begin{array}{l}\text { Categoría } \\
\text { animal } \\
\text { (encuestados) }\end{array}$ & $\begin{array}{l}\text { Nro. de marranas por } \\
\text { propietario }\end{array}$ & $\begin{array}{l}\text { Numero de } \\
\text { respuestas } \\
\text { (n) }\end{array}$ & $\begin{array}{l}\text { Venta promedio } \\
\text { al año }\end{array}$ & $\begin{array}{l}\text { Desviación } \\
\text { estándar }\end{array}$ \\
\hline Lechones & 0 & 3 & 19.3 & 11 \\
$(\mathrm{n}=36)$ & 1 a 5 & 15 & 41.5 & 44.9 \\
& 6 a 10 & 12 & 62.8 & 56 \\
& Más de 11 & 6 & 140.8 & 116.8 \\
Gorrinos & 0 & 4 & 13.5 & 13.9 \\
$(\mathrm{n}=38)$ & 1 a 5 & 16 & 18.9 & 25 \\
& 6 a 10 & 11 & 15.8 & 14.6 \\
\hline
\end{tabular}

Cuadro 3. Detalle del alimento que reciben los cerdos en las diferentes etapas de producción en granjas no tecnificadas de la zona de Lomo de Corvina, Villa el Salvador - Lima, Perú. 2010.

\begin{tabular}{llll}
\hline Etapa Productiva & Tipo de alimentación & $\begin{array}{l}\text { Numero } \\
(\mathrm{n})\end{array}$ & $\begin{array}{l}\text { Porcentaje } \\
(\%)\end{array}$ \\
\hline $\begin{array}{l}\text { Lechones } \\
(\mathrm{n}=49)\end{array}$ & Concentrado & 19 & 38.8 \\
& Restos de comida & 5 & 10.2 \\
& Mixto & 25 & 51 \\
$\begin{array}{l}\text { Gorrinos } \\
(\mathrm{n}=48)\end{array}$ & Concentrado & 2 & 4.2 \\
& Restos de comida & 35 & 72.9 \\
& Mixto & 11 & 22.9 \\
$\begin{array}{l}\text { Adultos } \\
(\mathrm{n}=48)\end{array}$ & Concentrado & 2 & 4.2 \\
& Restos de comida & 45 & 91.7 \\
\hline
\end{tabular}

Cuadro 4. Prácticas de manejo realizadas con los lechones al nacimiento en granjas no tecnificadas de la zona de Lomo de Corvina, Villa el Salvador - Lima, Perú. 2010 ( $\mathrm{n}=50)$

\begin{tabular}{lll}
\hline Práctica evaluada & $\begin{array}{l}\text { Respuestas afirmativas } \\
(\mathrm{n})\end{array}$ & $\begin{array}{l}\text { Porcentaje } \\
(\%)\end{array}$ \\
\hline Hierro & 49 & 96.1 \\
Desinfecta el ombligo & 16 & 31.4 \\
Corte de ombligo & 18 & 36.0 \\
Descolmilla & 32 & 64.0 \\
Coloca cama & 2 & 4.0 \\
Corte de cola & 6 & 12.0 \\
Coloca foco en corral & 2 & 4.0 \\
\hline
\end{tabular}


Cuadro 5. Frecuencia de enfermedades según época del año en granjas no tecnificadas de la zona de Lomo de Corvina, Villa el Salvador - Lima, Perú. 2010 (n=50).

\begin{tabular}{|c|c|c|c|c|}
\hline \multirow{2}{*}{$\begin{array}{l}\text { Signos clínicos o } \\
\text { enfermedad }\end{array}$} & \multicolumn{2}{|c|}{$\begin{array}{l}\text { Respuesta Afirmativa } \\
\text { para Verano }\end{array}$} & \multicolumn{2}{|c|}{$\begin{array}{l}\text { Respuesta Afirmativa } \\
\text { para Invierno }\end{array}$} \\
\hline & $\begin{array}{l}\text { Número } \\
\text { (n) }\end{array}$ & $\begin{array}{l}\text { Porcentaje } \\
(\%)\end{array}$ & $\begin{array}{l}\text { Número } \\
\text { (n) }\end{array}$ & $\begin{array}{l}\text { Porcentaje } \\
(\%)\end{array}$ \\
\hline No Reporta & 19 & 38 & 6 & 12.2 \\
\hline Cólera & 15 & 30 & 9 & 18.4 \\
\hline Diarrea & 13 & 26 & 5 & 10.2 \\
\hline Septicemia & 2 & 4 & 6 & 12.2 \\
\hline Respiratorio & 2 & 4 & 32 & 65.3 \\
\hline Problemas de Piel & 2 & 4 & 1 & 2 \\
\hline Golpe de Calor & 1 & 2 & 0 & 0 \\
\hline
\end{tabular}

Cuadro 6. Materiales usados en los corrales de las granjas de cerdos en Lomo de Corvina, Villa el Salvador - Lima, Perú. 2010.

\begin{tabular}{llll}
\hline Variable & Estrato de la Variable & $\begin{array}{l}\text { Número } \\
(\mathrm{n})\end{array}$ & $\begin{array}{l}\text { Porcentaje } \\
(\%)\end{array}$ \\
\hline $\begin{array}{l}\text { Cerco } \\
(\mathrm{n}=46)\end{array}$ & Madera & 43 & 93.5 \\
& Otros & 3 & 6.5 \\
$\begin{array}{l}\text { Pisos } \\
(\mathrm{n}=47)\end{array}$ & Tierra & 25 & 53.2 \\
& Cemento & 12 & 25.5 \\
& Tierra y cemento & 10 & 21.3 \\
& Madera & 17 & 36.2 \\
$\begin{array}{l}\text { Comederos } \\
(\mathrm{n}=47)\end{array}$ & Llanta & 11 & 23.4 \\
& Cemento + Madera & 8 & 17 \\
& Cemento & 5 & 10.6 \\
& Llanta + Madera & 4 & 8.5 \\
& Otras combinaciones & 2 & 4.2 \\
$\begin{array}{l}\text { Bebederos } \\
(\mathrm{n}=45)\end{array}$ & Madera & 20 & 44.4 \\
& No tiene & 18 & 40 \\
& Llanta & 3 & 6.7 \\
\hline & Otras combinaciones & 4 & 8.8 \\
\hline
\end{tabular}

Los registros de producción son documentos que ayudan a conocer el estatus productivo de la granja para poder valorar el costo-beneficio de la producción. En Lomo de corvina el $72.5 \%$ no lleva registros (productivos, reproductivos y de comercialización); el resto de productores solo lleva registros básicos.

Durante la comercialización de los cerdos solo el $7.8 \%$ de los criadores realiza la estimación del peso con balanza; mientras el $88.2 \%$ lo hace de forma visual (al ojo), el 2\% lo calcula de ambas maneras según la disponibilidad de balanza y solo el $2 \%$ no pesa a sus animales. El $88 \%$ de los criadores no tiene balanza propia mientras que el $11.8 \%$ restante cuenta con este instrumento.

La cantidad de lechones nacidos por parto en este sistema productivo fue de $10.1 \pm 1.9$ lechones. El 
número promedio de nacimientos al año por productor fue de $94.0 \pm 89.50$. En el cuadro 4 se pueden observar las prácticas de manejo que se realizan a los lechones al nacimiento y en el cuadro 5 la distribución de las enfermedades que afectan a este sistema productivo.

La condición de los corrales fue clasificada como buena, regular o mala. Fue buena cuando los corrales tenían piso de cemento, se encontraban limpios y tenían cercos, los bebederos y comederos se encontraban en buenas condiciones. Ello se observó solo en el $4.2 \%$ de los productores. Se consideró regular cuando el piso era de cemento pero no se eliminaban los desechos, los bebederos y comederos estaban sucios o en mal estado, esto fue observado en el $39.6 \%$ de productores y se consideró malo cuando el piso era de tierra, no contaban con bebederos o comederos o los que tenían estaban sucios lo que fue observado en el $56.3 \%$ de los casos. En el cuadro 6 se pueden observar las característica de los materiales utilizados en la crianza de los cerdos en Lomo de Corvina.

Para que se los trabajadores puedan realizar todas las actividades de la granja es necesario que tengan disponibilidad y accesibilidad a los servicios esenciales, tales como agua, desagüe y energía eléctrica. Al respecto tenemos que el $86.3 \%$ de los criadores mencionó que el abastecimiento de agua de su granja era a través del camión cisterna. Sólo el 3.9\% tuvo acceso al agua proveniente de la red pública del Servicio de Agua Potable y Alcantarillado de Lima SEDAPAL y el 9.8\% de los predios, utilizaba agua de pozo. En cuanto a la eliminación de excretas humanas el $86.3 \%$ contaba con letrinas. Solo el 2\% contaba con servicio de agua y desagüe. Mientras que el $11.7 \%$ de los predios no contaba con ningún sistema de eliminación de excretas humanas. Finalmente el $57.7 \%$ indico que contaba con servicio eléctrico en el predio, mientras que el $42.3 \%$ no contaba con este servicio. Finalmente, para la eliminación de excretas animales lo que usan con mayor frecuencia es el entierro (66.0\%) seguido del secado (21.3\%) y el resto de productores indican que botan las heces o usan otros métodos que no especificaron.

\section{DISCUSIÓN}

Los productores de cerdos incluidos en el estudio, fueron sensibilizados para participar en un programa de capacitación que se desarrolló en las granjas no tecnificadas ubicadas en la zona de Lomo de Corvina. Los datos consignados en el presente estudio representan la línea base de información, a partir de la cual se identificaron los principales problemas de esta población luego de lo cual se ofreció la capacitación y asistencia técnica para la crianza, comercialización y organización de los productores.

La participación en este tipo de crianza no tiene sesgo de género, por lo que la cantidad de propietarios, varones y mujeres, fue similar. Según la FAO, la participación de la mujer en este tipo de actividades contribuye al crecimiento económico de la familia (FAO, 2011). El ingreso familiar mensual es bajo para la mayoría de los propietarios; esto puede estar relacionado de manera indirecta al bajo nivel de instrucción; que no favorecería la mejora de la crianza basada en la autogestión. Sin embargo, en este grupo humano se ha podido evidenciar que existe desigualdad de género sobre todo en el área de educación, observándose una mayor prevalencia de mujeres con menor grado de instrucción. Esto trae como consecuencia una participación menos activa de las mujeres en la capacitación, debido a que al final de las mismas se entrega material escrito. Se ha podido observar que debido a la incapacidad de leer o de no comprender no se puede asegurar una mínima transferencia de tecnología, por lo que es imperante que el material a entregar sea en forma de manual pero con figuras para un mejor entendimiento del mismo.

En muchos casos los ingresos familiares suelen provenir exclusivamente de esta crianza, por ello la participación de la familia resulta importante, habiéndose encontrado un importante número de granjas donde toda la familia trabaja en la granja. De esta manera disminuyen los costos de producción y aparentemente mejoran los ingresos de la familia, debido a que no se valora económicamente la mano de obra de ellos.

En este sistema productivo se encuentran diferentes modos de crianza. Se puede observar que existen pobladores que tienen un sistema productivo completo (reproductores, crías y engorde); mientras que, otros se especializan en alguno de ellos. Según Rivera et al. (2007), esta especialización permite que el productor enfoque su atención y cuidados a un solo evento productivo lo que permite aumentar el movimiento de los animales y retornar el capital en menos tiempo. Algunos solo producen lechones, debido a que existe un mercado para esta categoría animal. En el primer caso se justificó la crianza de lechones para venta, por demandar una menor inversión de dinero en la alimentación (no había que buscar restos de comida para alimentar a los animales, solo le daban concentrado). Mientras los criadores de animales para 
engorde justificaban la compra de lechones destetados debido a que a esa edad la probabilidad de que los animales enfermen y mueran es menor.

La población de verracos es baja (34). Estos animales por lo general son alquilados para el servicio de las marranas, siendo desplazados a la granja donde se encuentra la hembra. Esta práctica favorecería la transmisión y diseminación de enfermedades.

En producciones porcinas tecnificadas el aislamiento (cuarentena) de todo animal que ingresa por primera vez a la granja es un componente crucial en el programa de bioseguridad. En estos casos los animales recién llegados deberían mantenerse separados del resto durante al menos 30 días, a fin de que el personal de la granja les haga un seguimiento. Evidentemente en el contexto en que se desarrolla este tipo de crianza, las condiciones para establecer medidas de bioseguridad son prácticamente nulas, por ello de presentarse alguna enfermedad es de esperar que esta ese diseminada en todas las granjas de la zona.

La etapa de comercialización, es el momento en la cual los productores obtienen el beneficio económico esperado. La mayor parte de los productores venden los animales vivos, directamente en la puerta de la granja a intermediarios informales, lo cual es desventajoso para ellos ya que los animales no son pesados, subvalorándose el verdadero precio. La venta en forma de carne también es común en la zona, sin embargo el sistema de matanza de los animales no se hace con las medidas higiénicas necesarias. Así tenemos que Castro (2007), indica que los procesos productivos se realizan sin prestar la debida atención a la manipulación, pudiendo aparecer brotes de ETA (enfermedades transmitidas por alimentos), que por lo inespecífico de la sintomatología en las personas y la falta de diagnóstico se desconoce la real magnitud de este tipo de problemas. Lo mismo ocurre con los residuos biológicos (como antimicrobianos o agroquímicos) que podrían acumularse en las canales de los cerdos.

En Lomo de Corvina la venta de los animales por lo general se realiza cuando el productor tiene necesidad de dinero en efectivo, y no cuando los animales han logrado el peso de venta al mercado. Esto concuerda con lo señalado por Rivera et al. (2007) quien indica que las causas por las cuales los productores venden sus animales son: emergencias económicas (53\%), baja producción $(20 \%)$, enfermedad (11\%) y vejez $(9 \%)$.
Sin embargo, los productores en general no conocen con cierta exactitud la ganancia obtenida por la venta de sus animales. Esto se debe a que no tienen registros de producción y tampoco valorizan su trabajo (mano de obra). Pudiéndose inferir que la administración de la granja se hace de manera empírica, debido a que no se llevan registros de ingresos y egresos.

Con respecto a la alimentación, esta varía según la etapa productiva. Así tenemos que la mayoría de lechones reciben concentrado o alimento mixto (restos de comida y concentrado), debido a que son considerados más susceptibles de sufrir problemas entéricos. Los gorrinos reciben generalmente resto de comida o una alimentación mixta. Los adultos son alimentados en su mayoría con restos de comida ya que solo requieren una dieta de mantenimiento. Además los productores consideran que la inclusión del concentrado en la dieta de los animales solo debe ser en casos especiales. Es así que cuando los productores quieren vender sus animales en el corto plazo le adicionan concentrado a la dieta; a fin de acortar el tiempo de ganancia de peso y tener un animal que le genere mayores ingresos económicos, lo que en muchos casos consiguen aunque por lo general no tienen información de este logro. En general, para estas dos últimas categorías, se puede decir que el criador considera que el gasto del concentrado es elevado, y no puede mantener este costo hasta el momento de la saca.

El alimento que se ofrece a los animales y que proviene de los residuos debe de ser tratado térmicamente. Esta práctica se encuentra normada en el anteproyecto de guía técnica sanitaria para las crianzas de cerdos, en el que se menciona que los restos de alimentos destinados a la alimentación de esta especie, debe ser sometida a tratamiento térmico $\left(100^{\circ} \mathrm{C}\right.$, ebullición) por al menos 5 minutos desde que empieza la ebullición, pudiéndose recién usar para la alimentación animal. Con esta práctica se trata de higienizar el alimento que se le ofrece al animal.

Se debe de considerar que los procesos de descomposición favorecen la preservación de huevos de parásitos y microorganismos que pueden ser el origen de numerosos trastornos infecciosos, principales gastrointestinales y dérmicos. Por ello, el tratamiento térmico busca reducir el riesgo de transmisión de estas enfermedades a los animales. Sin embargo, Santandreu et al. (2002) señala que los efectos del tratamiento térmico se ven afectados negativamente cuando se tratan volúmenes grandes de 
alimento. Esto debido a que la temperatura obtenida no permite una cocción uniforme, quedando parte de la masa sin tratar.

El material utilizado como combustible fue leña (madera) y el envase para cocinar fueron generalmente cilindros. Estos son obtenidos durante la faena de reciclaje, con lo que los costos asociados al tratamiento térmico de los alimentos, disminuyen.

La falta de ayuda profesional se debe a la situación de pobreza de la mayor parte de los criadores. Esto lleva a que los animales se encuentren expuestos a enfermedades que pueden producir alta morbilidad y mortalidad, afectando los ingresos económicos del sistema productivo y la salud de las personas en caso de enfermedades potencialmente zoonóticas. Dentro de los factores asociados a estos tenemos: la falta de control sanitario, la utilización de residuos sin tratamiento para la alimentación, el acceso limitado a medicamentos veterinarios, la desinformación sobre las principales enfermedades de los animales y la ausencia de seguimiento de algún organismo regulador (Municipalidad, Ministerio de Agricultura, Ministerio de Salud).

Con respecto al programa sanitario, la mayor parte de criadores $(90 \%)$ aplica vacuna contra el cólera porcino. Sin embargo, hay un $10 \%$ de criadores que no realizan esta acción preventiva, aun cuando la vacunación contra esta enfermedad en los periodos de engorde y en zonas con reactivaciones periódicas, es obligatoria debiéndose realizar con una frecuencia de seis meses.

Muy pocos llevan registros productivos o reproductivos escritos (fecha de nacimiento, número de lechones por marrana, fecha de monta, cubrición o servicio de la marrana, peso a distintas edades, mortalidad, etc.), con lo que resulta difícil evaluar los parámetros de la granja e implementar y evaluar algunas intervenciones aisladas. Tampoco llevan un registro del dinero que invierten, los costos que se producen (compra de medicina o alimento, cantidad de alimento proporcionado por corral) por lo que se desconoce la rentabilidad de la granja. Esto se refleja en el desconocimiento de la ganancia de peso en los animales por kilogramo de alimento suministrado. La ganancia de peso y el peso final no es conocida principalmente por la ausencia de balanza.

En la mayoría de los casos, los corrales tienen piso de tierra, estos fácilmente se hacen huecos, llenándose de agua y desperdicios, convirtiéndose en un foco de infección permanente que puede producir muchas enfermedades. En otras, los corrales tienen piso de cemento, sobre todo el área de maternidad. Los comederos son generalmente de madera, o de cemento. La mayoría de los bebederos son de madera; aunque, el $40 \%$ de los criadores no colocan bebederos en el corral, esto se debe a que no dan agua a sus animales ya que consideran, que ello es suministrado con el alimento (sopa).

Se observado que los criadores no cuentan con un óptimo programa de manejo de lechones (cuadro 6), por lo que se puede inferir que causas como hipotermia, hipoglucemia, canibalismo, infecciones y otras pueden presentarse. Así por ejemplo, se ha observado que los lechones que son aplastados por la cerda por lo general tienen un bajo peso y están muy débiles, con síntomas de hipoglucemia y enfriamiento, es decir que no cuentan con la energía suficiente para evadir a la madre cuando ésta se acuesta o cambia de postura. En época de invierno los problemas respiratorios aumentan considerablemente, esto puede deberse a que el clima en la zona es bastante frío y húmedo, los criadores no cuentan con corrales bien estructurados ni limpios Según Radostits et al (2002), la baja temperatura y la humedad, modifican la penetración en los pulmones de patógenos primarios y secundarios, al alterar el tamaño de partículas de aerosol infectadas y el mecanismo protector de las vías respiratorias. Así, se altera la sedimentación y la concentración de partículas infectadas que van por el aire. Los problemas de diarreas son frecuentes durante todo el año predominado en verano, esto probablemente se deba a que la elevación de la temperatura ambiental favorece la fermentación del alimento el cual por lo general está almacenado a la intemperie debido a que no existe un sistema de almacenamiento y conservación.

En cuanto a la eliminación de las heces de los cerdos, tenemos que por lo general los criadores prefieren enterrarlas. Sin embargo, se pudo observar que más del $80,4 \%$ de los productores no realiza un manejo higiénico de estas excretas, por lo que preocupa la falta de alternativas de manejo de los residuos sólidos orgánicos generados, dado que pueden representar un riesgo para la salud de los animales y las personas. Mariscal (2007), menciona que el principal problema ocasionado por las excretas es la contaminación química, debida a la excreción de grandes cantidades de nitrógeno (en forma de nitratos), fósforo y potasio, además de los físicos (materia orgánica y sólidos en 
suspensión) y el olor ocasionado por una gran cantidad de compuestos orgánicos volátiles entre ellos el amoniaco.

Mariscal (2007), también señala que para minimizar el impacto ambiental que generan las excretas se deben reducir al mínimo los residuos en cada etapa de producción. Para ello, es necesario desarrollar nuevas prácticas y mejorar los procedimientos con el fin de mejorar la utilización de los nutrientes y de esta manera reducir las emisiones al medio ambiente. Por otro lado, Rivera (2007) menciona que la inadecuada eliminación de excretas favorece el desarrollo de plagas (ratas, cucarachas, moscas), lo que es más evidente en crianzas donde la limpieza se realiza con poca frecuencia.

La falta de agua representa un gran problema para cumplir con las normas técnico-sanitarias para la crianza de cerdos. La mayor parte $(86.5 \%)$ se abastece de agua de los camiones cisterna lo que crea dudas en cuanto a la calidad de estas aguas, ya que por lo general son aguas no potabilizadas; además elevan los costos en comparación al agua obtenida de la red pública.

En cuanto a la disposición de excretas humanas, la mayoría de granjas cuentan con letrina. Sin embargo, algunas de estas no cuentan con ningún sistema o plan de evacuación de heces humanas. Esto puede representar un riesgo para los animales ya que existen algunas parasitosis como la cisticercosis que pueden ser transmitidas a través de las heces humanas, por lo que es necesario considerar que muchos de los criadores no son de Lima con lo cual podría existir el riesgo de que algún criador o trabajador sea portador de la Taenia solium.

La electricidad es un servicio que es necesario en las granjas de cerdos, sobre todo para lagunas etapas en las cuales se necesitan aumentar la temperatura como en el área de maternidad. Sin embargo no todos los pobladores cuentan con acceso a esta, la falta de suministro de electricidad imposibilita el desarrollo social, cultural y económico de este parque porcino; considerando que los trabajos de llenado de registros y la lectura de información técnica se suelen hacer de noche, con lo que la ausencia de luz es una limitante para el desarrollo de estas prácticas y para el acceso a información en términos generales (radio, TV).

De acuerdo a los resultados, la mejora de este sistema de crianza requiere de apoyo externo; el cual puede ser dado a través de capacitaciones en términos normativos, manejo sanitario, reproductivo y alimenticio, y mercadeo. Asimismo, los resultados del estudio permiten identificar las principales deficiencias de este proceso productivo y a partir del mismo se pueden diseñar estrategias específicas de intervención para superarlas.

\section{Correspondencia}

Daphne Ramos Delgado

dramosd@unmsm.edu.pe

\section{AGRADECIMIENTOS:}

Los autores agradecen la colaboración y facilidades brindadas para la realización de este trabajo a todos los criadores de cerdos de la zona Lomo de Corvina en el distrito de Villa El Salvador, Lima.

\section{REFERENCIAS BIBLIOGRÁFICAS}

1. Arce B, Alegre J, Escudero D, Prain G, Saenz J. 2007. Crianza de cerdos en zonas urbanas: diagnostico y propuesta municipal de sistema de manejo en el distrito de Lurigancho Chosica, Lima-Perú. En: Castro G. (compilador) Porcicultura Urbana y Periurbana en Ciudades de América Latina y el Caribe. IPES Promoción del Desarrollo Sostenible. Lima-Perú. Cuaderno de Agricultura Urbana $\mathrm{N}^{\circ} 1.59 \mathrm{p}$.

2. Castro G. (compilador) 2007. Porcicultura Urbana y Periurbana en Ciudades de América Latina y el Caribe. IPES Promoción del Desarrollo Sostenible. Lima-Perú. Cuaderno de Agricultura Urbana $\mathrm{N}^{\circ} 1.59 \mathrm{p}$.

3. Castro G, Lozano A. 2009. Desafíos y oportunidades para la Ganadería Urbana y Periurbana en ciudades de América Latina y El Caribe. Cuaderno de Agricultura Urbana $\mathrm{N}^{\circ}$ 7. IPES Promoción del Desarrollo Sostenible. Lima, Perú. 44 p.

4. FAO. 2011. El estado mundial de la agricultura y la alimentación: Las mujeres en la agricultura. [Internet] [Acceso 15 de abril del 2011] Disponible en: http:// www.fao.org/docrep/013/i2050s/i2050s.pdf

5. Fernández A, Sánchez M. 2007. Guía para la gestión integral de los residuos sólidos urbanos. Organización de las Naciones Unidas para el Desarrollo Industrial (ONUDI) [Internet] [Acceso 15 de noviembre del 2010] Disponible en: http://www.unido.org/ fileadmin/import/72852_Gua_Gestin_Integral_de_ RSU.pdf

6. Hernández J, Rebollar S, Rojo R, García J, Guzmán E, Martinez J, Díaz M. 2008. Rentabilidad privada de las granjas porcinas en el sur del estado de México. Universidad y Ciencia 24(2):117-124

7. Kalinowski J. 1996. Notas sobre el transporte de cerdos a camal. Boletín de la Asociación Peruana de 
Porcicultores 5: 5-6.

8. Mariscal, G. 2007. Tratamiento excretas cerdos. Capítulo 7. In: Reporte de la Iniciativa de la Ganadería, el Medio Ambiente y el Desarrollo (LEAD) - Integración por Zonas de la Ganadería y de la Agricultura Especializadas (AWI) - Opciones para el Manejo de Efluentes de Granjas Porcícolas de la Zona Centro de México. Departamento de Agricultura de la FAO. Instituto Nacional de Investigaciones Forestales, Agrícolas y Pecuarias (México). Universidad Nacional Autónoma de México (México), Swiss College of Agriculture (Switzerland), y Coordinado por la FAO. CENID. [Internet] Disponible en: www.fao.org/wairdocs/ LEAD/X6372S/x6372s08.htm

9. Rivera J, Losada H, Cortes J, Grande, Vieyra J, Castillo A, Gonzalez R. 2007. Cerdos de traspatio como estrategia para aliviar pobreza en dos municipios conurbados al oriente de la Ciudad de México. Livestock Research for Rural Development 19: artículo 96.

10. Radostits O, Gay C, Blood D, Hincheliff K. 2002. Medicina Veterinaria. MacGraw Hill, Madrid, España. Tomo 1. 1206 p.
11. Rodríguez D, Anchieri D, Tommasino H, Vitale E, Moreira R, Castro G, Lozano A, López C. 2003. Tratamiento de residuos sólidos orgánicos domiciliarios para la alimentación de cerdos. [Internet] [Acceso 15 de diciembre del 2010] Disponible en: http://www.bvsde.paho.org/bvsacd/ cd29/cerdos.pdf

12. RuizA. 2010. Mejora de las condiciones de vida de las familias porcicultoras del Parque Porcino de Ventanilla, mediante un sistema de biodigestión y manejo integral de residuos sólidos y líquido. Tesis Doctoral de Ingeniería Química. Universidad Ramón Llull, España. 211 p.

13. Santandreu A, Castro G, Ronca F. 2002. La cría de credos en asentamientos irregulares. Cuadernos de Montevideo Rural $N^{\circ}$ 2. Intendencia Municipal de Montevideo, Uruguay. 40p.

14. Zarate R. 2006. Diagnostico estático de la crianza porcina no tecnificada de la zona agropecuaria del distrito de Villa el Salvador, Lima Metropolitana. Tesis para optar el título de Ingeniero Zootecnista. Universidad Agraria de La Molina, Lima Perú. 50 p.

Recibido: 26/11/2014

Aceptado: 17/12/2014 